Muropeptide aus den Sphäroplasten-Membranen herauszuholen waren. Vielleicht wirken diese für die zelleigenen Hydrolasen unzugänglich bleibenden Mureinreste in der Sphäroplasten-Membran als „Primer" 15, 16, die eine Wiederaufnahme der Mureinsynthese nach Entfernung des Penicillins und damit die Reversion solcher Sphäroplasten in die stabile Stäbchenform ${ }^{17}{ }^{18}$ ermöglichen.

Eine genaue Bilanz über den Verbleib der radioaktiven DAP bei der Umwandlung einer Coli-Zelle in einen Sphäroplasten läßt sich aus mehreren Gründen nicht aufstellen. Erstens wird wohl ein kleiner Teil der angebotenen DAP in Lysin umgewandelt $^{19,20}$; zweitens enthalten die radiomarkierten

15 K. McQuillen, in: J. C. Gunsalus u. R. Y. Stanier (Editors), The Bacteria, Vol. I, Academic Press, 1960.

16 C. LaRk u. K. G. Lark, Biochim. biophysica Acta [Amsterdam] 49, 308 [1961].

17 J. Lederberg, Proc. nat. Acad. Sci. USA 42, 574 [1956].

18 K. Liebermeister u. E. Kellenberger, Z. Naturforschg. 11 b, 200 [1956].
Zellen, wie wir ${ }^{21}$ und auch andere Autoren ${ }^{22}$ feststellten, zu Beginn der Penicillineinwirkung etwa $10 \%$ der gesamten DAP als löslichen Pool, drittens schließlich lysiert ein wechselnder Prozentsatz der Zellen während der Umwandlung in Sphäroplasten, so daß hierdurch und wahrscheinlich auch durch direktes Herausdiffundieren aus intakten Sphäroplasten ein Teil der DAP-haltigen Peptide ins Nährmedium übertritt ${ }^{21}$. Eine quantitative Wiedergewinnung und Charakterisierung ist dann wegen des komplexen Mediums praktisch unmöglich. Auf dem von uns eingeschlagenen Wege glauben wir, trotzdem zuverlässige Daten zum besseren Verständnis der Penicillinwirkung, die noch lange nicht endgültig geklärt ist, erhalten zu haben.

19 D. L. Dewey u. E. Work, Nature [London] 169, 533 [1952].

20 B. D. Davis, Nature [London] 169, 534 [1952].

21 U. Schwarz, Dissertation, Tübingen (1963).

22 A. Tomasz u. E. Borek, Biochemistry 1, 543 [1962].

\title{
Eigenschaften eines Homogenisats von Hefe ${ }^{1}{ }^{1}$
}

\author{
P. Ohlmeyer, G. Müller und U. Heun \\ Leibniz-Kolleg der Universität Tübingen \\ (Z. Naturforschg. 20 b, 157-163 [1965] ; eingegangen am 13. November 1964)
}

\begin{abstract}
Tropfen eines Homogenisats von Bäckerhefe wurden eingedunstet, wieder bewässert und nach einem oder zwei Tagen mit Hefekochsaft verdünnt. Drei oder zwei Tage später fanden sich in zahlreichen Ansätzen dieser Art Zellkulturen, die zumeist von Bäckerhefe verschieden waren.

Auch das Homogenisat von roter Hefe lieferte in diesem Prozeß Kulturen, neben roten auch Zellen anderer Art.

Neue Zellen fanden sich ferner, wenn das Homogenisat von Bäckerhefe mit Glucose oder Glucose/Kochsaft geschüttelt wurde. Die Schüttelansätze erbrachten nur eine sehr geringe Zahl von Zellkulturen, wenn die Hefe in Gegenwart von Nucleasen homogenisiert worden war.
\end{abstract}

Im ersten Teil dieser Mitteilung beschreiben wir die verschiedenen Zellarten, die in unseren Ansätzen auftraten, sodann die Ansätze selbst und ihr zahlenmäßiges Ergebnis. Es folgt die Darstellung der Kontrollversuche, der Ansätze mit den Nucleasen und der Versuche mit roter Hefe. - Der zweite Teil bringt, in der Gliederung des ersten, Anmerkungen zur Methodik.

\section{I}

\section{a) Zellarten}

Mit Sicherheit konnten wir in der Reinkultur und unter dem Mikroskop 9 Zellarten unterscheiden.
Systematisch wissen wir nur 2 von ihnen einzuordnen; alle tragen im folgenden die Bezeichnung, die sie im Gang der Arbeit erhalten haben. Wir unterscheiden sie nach den Eigenschaften, die in Tab. 1 angegeben sind.

Weiteres zur Charakterisierung der Zellen trug die Beobachtung ihres Wachstums unter dem Deckglas und in der Nährlösung an der Luftgrenze (Kahmbildung) bei. - Über Rhodotorula haben wir einiges bereits mitgeteilt ${ }^{1}$; aus ihrem Homo-

1 1. Mitt. Z. Naturforschg. 20 b, 58 [1965] ; vorl. Mitt. Z. Naturforschg. 19 b, 1087 [1964]. 


\begin{tabular}{|c|c|c|c|c|c|}
\hline Bezeichnung & $\begin{array}{c}\text { mittlere } \\
\text { Größe } \\
{[\mu]}\end{array}$ & $Q_{\mathrm{CO}_{2}}^{\mathrm{N}_{2}} / Q_{\mathrm{O}_{2}}$ & $\begin{array}{c}t_{\mathrm{v}} \\
{[\text { Min. }]}\end{array}$ & $\begin{array}{c}\text { Vermehrung } \\
\text { ohne } \mathrm{O}_{2} ?\end{array}$ & Bemerkungen \\
\hline $\begin{array}{c}\text { B } \\
\text { (Bäckerhefe) }\end{array}$ & $7,5 \cdot 6$ & $\frac{204}{62}=3,3$ & 100 & $\mathrm{ja} ; t_{\mathrm{v}}=101$ & $\begin{array}{l}\text { morphologisch } \\
\text { ähnlich } P\end{array}$ \\
\hline $\mathrm{Z}$ & $5,5 \cdot 5,5$ & 0,04 & 95 & nein & \\
\hline A & $\sim 13 \cdot 6$ & 0,37 & 140 & nein & $\begin{array}{l}\text { mehrfache, } \\
\text { Sprossung, } \\
\text { Neigung zu } \\
\text { Pseudomycel }\end{array}$ \\
\hline $\mathrm{S}$ & $8,5 \cdot 3,5$ & 0,4 & 142 & nein & \\
\hline $\mathrm{X}$ & $3,5 \cdot 2,5$ & $\frac{96}{59}=1,6$ & 66 & $t_{\mathrm{v}}>1000$ & \\
\hline $\mathrm{L}$ & $4,5 \cdot 3,5$ & $\frac{180}{77}=2,3$ & 56 & - & \\
\hline $\begin{array}{c}\mathrm{Y} \\
\text { (Rhodotorula) }\end{array}$ & $5 \cdot 3,5$ & $\frac{1,26}{51}=0,025$ & 121 & nein & rot \\
\hline $\mathrm{G}$ & $4 \cdot 2,2$ & $\frac{334}{124}=2,7$ & 63 & - & \\
\hline $\mathrm{P}$ & $10,5 \cdot 7,5$ & $\frac{193}{39}=5$ & 77 & $t_{\mathrm{v}}=77$ & ähnlich B \\
\hline SS & - & - & - & - & s. Text \\
\hline
\end{tabular}

Tab. 1. Charakteristik der Zellarten $\left(t_{\mathrm{V}}=\right.$ Verdoppelungszeit $)$.

genisat vor allem erschien sehr oft SS, deren Zelle klein wie die kleinsten beginnt, wächst, zur Luft strebt und wie ein Schimmelpilz pigmentierte Sporen bildet. - Oft sahen wir auch die vor kurzem beschriebenen atmenden Teilchen ${ }^{1}$; in Statistiken haben wir sie nicht aufgenommen. - Näheres zu Tab. 1 unter Methodik.

\section{b) Homogenisate}

1,0 g Bäckerhefe wurde mit $2 \mathrm{~g}$ Sand 5 Min. in der Reibschale zerrieben, in dieser Mischung mit $5 \mathrm{ml}$ Wasser aufgenommen und 7-mal für 5 Min. bei $1200 \mathrm{~g}$ in der Weise zentrifugiert, daß jeder Überstand in ein neues Glas gebracht wurde. Die letzten 5 Zentrifugengläser waren innen gefettet. Das Homogenisat ist trüb; es wurde bei $10^{\circ}$ aufbewahrt.

Ein zweites Verfahren, das in der vorliegenden 2. Mitteilung für rote Hefe ausschließlich angewandt wurde, ist im methodischen Teil beschrieben.

\section{c) Tropfenmethoden}

1. Wir tropften je $15 \mu$ l frisches Homogenisat in ca. 40 Glasschälchen, die in einer Deckelschale in zwei Kreisen um ein größeres flaches Gefäß angeordnet waren. Das flache Gefäß enthielt etwas Calciumchlorid. Bei $28^{\circ}$ war das Homogenisat in $5 \mathrm{Stdn}$. unter $1 \mathrm{mg}$ eingeengt. Das Calciumchlorid wurde in
Wasser gelöst oder entfernt, und auf den Boden der Schale gebrachtes Wasser bewirkte, daß die Tropfen sich wieder benäßten. Nach 24 Stdn. nahmen wir einen Teil des Tropfens mit $10 \mu \mathrm{l}$ Hefekochsaft in der Pipette hoch und übertrugen ihn in eine frisch aufgebrochene Ampulle, die $\theta, 1 \mathrm{ml}$ Kochsaft enthielt; oder wir brachten $60 \mu \mathrm{l}$ Kochsaft auf den Tropfen. Nach 2 Tagen wurde mikroskopiert.

2. Unterschiede gegenüber Methode 1: Kein Calciumchlorid, 11 oder $5 \mu$ l Homogenisat, 1 Tag, Wasser auf den Boden der Schale, 2 Tage, 0,1 ml Kochsaft/Glucose/Streptomycin auf den Tropfen, 2 Tage, mikroskopische Untersuchung.

3. Die Kulturen, die nach der ersten Tropfenmethode aus 11 Hefesendungen $(25.5$ bis 24.9 . 1962) erhalten wurden, sind in Tab. 2 verzeichnet.

\begin{tabular}{|c|c|c|c|c|c|c|c|}
\hline \multirow{2}{*}{$\begin{array}{c}\text { Zahl der } \\
\text { Tropfen }\end{array}$} & \multicolumn{7}{|c|}{ Zahl der Kulturen } \\
\cline { 2 - 8 } & B & Z & A & S & X & L & Y \\
\hline 727 & 0 & 41 & 6 & 22 & 23 & 4 & 2 \\
\hline & & & & & & $\sum: 98$ \\
\hline
\end{tabular}

Tab. 2. Zellkulturen aus Tropfen von Homogenisat.

Die Kulturen differierten beträchtlich in der Zahl der Zellen. Maxima lagen bei $10^{4} / \mu \mathrm{l}$; positiv nennen wir noch ein Minimum von $10 / \mu$, aber nur dann, wenn Sprosse zu sehen sind. - In Tab. 2 sind 


\begin{tabular}{|c|c|c|c|c|c|c|c|c|c|c|}
\hline \multirow{2}{*}{ Tropfen } & \multicolumn{4}{|c|}{ Kulturen } & \multicolumn{2}{c|}{ Kontrollen } & \multicolumn{2}{c|}{ Gemisch-Ampullen } \\
\cline { 2 - 5 } & $\mathrm{Z}$ & $\mathrm{S}$ & $\mathrm{X}$ & $\mathrm{SS}$ & $\mathrm{Y}$ & & positiv & negativ & positiv & negativ \\
\hline 116 & 1 & 3 & 1 & 4 & 83 & 8 & 3 & 56 & 0 \\
\hline
\end{tabular}

Tab. 3. Zellen aus Tropfenansätzen zweiter Art.

20 zweifache und je 4 drei- und vierfache Kulturen enthalten. (Wegen der Auftrennung von Mischkulturen s. unten.) - Aus dem Homogenisat von Bäckerhefe ist, wie man sieht, keine Bäckerhefe hervorgegangen.

4. Für die zweite Tropfenmethode haben wir auch nach dem zweiten Verfahren homogenisiert; entscheidend für die Ausbeute an Kulturen ist hier aber, daß der wieder bewässerte Tropfen einen Tag länger verweilt. Jedes der 3 Homogenisate (aus 3 Hefesendungen) lieferte außer den 40 Tropfen zu $11 \mu \mathrm{l}$ 20 Portionen zu $22 \mu$, die für 5 Tage mit $0,5 \mathrm{ml}$ Kochsaft/Streptomycin in Ampullen aufbewahrt wurden. $\mathrm{Zu}$ jedem Versuch mit 40 Tropfen gehören ferner 3 Ampullen, die je $1 \mathrm{ml}$ des Gemisches Kochsaft/ Glucose/Streptomycin enthalten, mit dem die Tropfen nach 3 Tagen versetzt wurden (Tab. 3 ).

In den Versuchen zu Tab. 3 hat das Homogenisat von Bäckerhefe in 92 Kulturen aus 116 Tropfen wiederum keine Bäckerhefe geliefert. Beim Zerreiben verschonte Zellen dieser Art fanden sich in 3 Kontroll-Ampullen.

Diese Kontrollen zeigen zuverlässig an, ob das Homogenisat verschonte Zellen enthält. Werden nämlich $k$ Teilchen auf $n$ gleiche Volumina so verteilt, daß alle Möglichkeiten der Verteilung gleiche Wahrscheinlichkeit besitzen, dann ist die Wahrscheinlichkeit für den Zustand, in dem $i$ Teilchen in einem Probevolumen angetroffen werden, nach einer Rechnung von B. Artmann

$$
W=\frac{\left(\begin{array}{l}
k \\
i
\end{array}\right) \cdot(n-1)^{k-i}}{n^{k}} .
$$

Für 80 Zellen in 100 Volumina errechnen sich angenähert 45 leere Volumina. - Wir schüttelten Serien mit insgesamt 500 in der Verdünnung gezählten Zellen der Arten Z, A, X, L und Y (von dieser 105 Zellen) in ingesamt $312 \mathrm{ml}$ Kochsaft aus Bäckerhefe und schmolzen je $0,5 \mathrm{ml}$ dieser Suspension in 624 Ampullen ein. Nach 3 Tagen der Aufbewahrung bei Zimmertemperatur wurden 305 Ampullen $(=49 \%)$ leer gefunden; in den Serien mit der roten
Art Y waren 51 (=48,5\%) leer. - Das Verteilungsverfahren ist also sehr zuverlässig; wir benutzen es deswegen auch zur Auftrennung von Mischkulturen.

\section{d) Schüttelansätze}

Für diese Versuche (Tab. 4) wurde Homogenisat aus Bäckerhefe 24 Stdn. oder länger, höchstens 72 Stdn., bei $10^{\circ}$ aufbewahrt. Die Ansätze enthielten je 0,2 ml Homogenisat, 20-proz. Glucose, Kochsaft; $0,4 \mathrm{ml}$ Wasser; ca. 3,5 ml Luft. Sie wurden durchweg $17 \mathrm{Stdn}$. bei $28^{\circ}$ geschüttelt.

\begin{tabular}{|c|c|c|c|c|c|c|}
\hline \multirow{2}{*}{$\begin{array}{c}\text { Zahl der } \\
\text { Ansätze }\end{array}$} & \multicolumn{5}{|c|}{ Anzahl der Kulturen } \\
\cline { 2 - 7 } & P oder B & Z & A & S & X & L \\
\hline 83 & 32 & 47 & 7 & 16 & 2 & 22 \\
\hline & & & & & & $\sum: 126$ \\
\hline
\end{tabular}

Tab. 4. Zellkulturen aus Schüttelansätzen.

Die Schüttelansätze ergaben ähnlich den Tropfen auch Mischkulturen. In Tab. 4 sind 23 zweifache, 14 dreifache und 3 vierfache Kulturen enthalten. Die einander ähnlichen Arten $\mathrm{P}$ und $\mathrm{B}$ wurden als vorkommend gesichert, aber nicht in jedem Gefäß identifiziert.

Da hier mit Nährlösung von definiertem Volumen geschüttelt wurde, ermöglicht die im Aliquot gefundene Anzahl der Zellen $\left(A_{\mathrm{t}}\right)$ eine Umrechnung auf ihre Anzahl bei Beginn des Schüttelns $\left(A_{0}\right)$. Denn wir kennen die Verdoppelungszeit $\left(t_{\mathrm{v}}\right)$ einer Zellart und wissen von dem Beispiel Bäckerhefe (vgl. Methodik), daß $t_{\mathrm{v}}$ von den Ernährungsbedingungen recht unabhängig ist. Ferner ist die Zeit $(t)$ bekannt, die für Vermehrung zur Verfügung stand. Aus $A_{\mathrm{t}}=A_{0} \cdot e^{0,693 \cdot t / t_{\mathrm{v}}}$ errechnen sich dann für die Kulturen aus Tab. 4 die in Tab. 5 zusammengestellten Werte für $A_{0}$; wir nennen sie die reduzierten Zellenzahlen.

\begin{tabular}{|c|c|c|c|c|c|}
\hline$A_{0}$ & $>100$ & $21-100$ & $6-20$ & $1-5$ & $<1$ \\
\hline $\begin{array}{c}\text { Zahl der } \\
\text { Kulturen }\end{array}$ & 11 & 10 & 16 & 60 & 29 \\
\hline
\end{tabular}

Tab. 5. Reduzierte Zellenzahlen. 
Zur Deutung von Tab. 5 setzen wir zunächst willkürlich an, daß das frisch hergestellte Homogenisat keine Zellen enthalten hat. Dann gilt für den Fall $A_{0}>1$, daß bei Beginn des Schüttelns entweder Zellen vorlagen, die im Homogenisat bei $10^{\circ}$ erschienen waren, oder daß keine Zellen vorlagen, vielmehr erst später, während des Schüttelns, erschienen. Für den Fall $A_{0}<1$ (der 29-mal eingetreten ist) gilt allein, daß die Zellen später erschienen.

Nun wird die Frage, ob und in welcher Anzahl ein frisches Homogenisat Zellen enthalten hat, durch die Statistik beantwortet, die mit den Kontrollen zu Tab. 3 begann und im folgenden fortgesetzt wird.

\section{e) Prüfung des Homogenisats auf Gehalt an Zellen}

In diesem Abschnitt geben wir Überlegungen und Versuche wieder.

1. In Tab. 5 kommen Ansatz-Serien vor, für die aus dem gleichen, gut umgerührten Homogenisat je $0,2 \mathrm{ml}$ in mehrere Schüttelgefäße gebracht wurden. Es werden dann aber stark differierende Werte für $A_{0}$ beobachtet. Fassen wir die Ansätze zusammen, die bei gleichem Homogenisat stark differieren, dann stehen 11 Gefäße mit $A_{0}>100$ (Durchschnitt 265) 13 Gefäßen mit $A_{0}$ zwischen $<1$ und 5 gegenüber. Nun ist es nahezu ausgeschlossen, daß mit je $0,2 \mathrm{ml}$ aus einer Flüssigkeit mit statistisch verteilten Zellen so oft so viele und so oft so wenige Zellen entnommen werden. Diese Überlegung spricht sehr dafür, daß die Zellen erst während des Schüttelns aufgetreten sind.

2. Wenn ein Homogenisat von Bäckerhefe fremde Zellen enthält, so können sie aus der Preßhefe stammen. In Vermehrungsversuchen, die in sehr großer Anzahl ausgeführt wurden, haben wir sie auch gesehen, aber nur L und Y, je einmal in Deckglaskulturen.

3. Ist Preßhefe mit X im Verhältnis $125000: 1$ kontaminiert, dann ist $\mathrm{X}$ folgendermaßen zu entdecken: In kugeligen Gefäßen mit einem konischen Ansatz an der tiefsten Stelle wurden 1,6 ml Nährlösung unter $13 \mathrm{ml} \mathrm{Luft} \mathrm{geschüttelt.} \mathrm{Die} \mathrm{Lösung} \mathrm{ent-}$ hielt in 4 Gefäßen (a) nach Rechnung je 500000 Zellen B, in 5 Gefäßen (b) außerdem je 4 Zellen X. Die größere B fällt dann rascher in den Konus und wird dort schlecht ernährt; $\mathrm{X}$ wächst rascher im Verhältnis $100: 66$ (vgl. Tab. 1). Nach 18 Stdn. fanden wir in 4 Gefäßen (b) außerhalb des Konus B und $\mathrm{X}$ im Verhältnis $67: 1$; in den 4 Gefäßen (a) sahen wir weder $\mathrm{X}$ noch eine andere rasch wachsende Zelle.
Die Kontamination der Preßhefe war also sicherlich sehr gering.

4. Von $98+92+126=316$ Kulturen enthielten 284 mit Sicherheit keine Zellen B (Tab. 2, 3, 4). Will man dieses Verhältnis mit Zellen aus der Preßhefe erklären, dann muß man annehmen, daß allein durch das Verfahren (mit $1 \mathrm{~g}=\mathrm{ca} .10^{10}$ Zellen B) das ursprüngliche Zahlenverhältnis der Arten immens verändert wurde. Es hieße, daß fremde Zellen sehr viel schlechter zerrieben und verschonte fremde Zellen sehr viel schlechter abzentrifugiert werden. Gegen beides spricht die Vermutung sehr stark.

5. Wir prüften das frische Homogenisat auf Zellen, indem wir zunächst sicherstellten, daß fremde Arten sich darin vermehren; dies aber mit $1: 5$ verdünntem Homogenisat, das wir im Hinblick auf das Auftreten von Zellen für „totverdünnt“ halten durften. In 0,5 ml waren je 5 Zellen eingesät (Tab. 6, A). Parallel liefen Ansätze ohne Einsaat. Mikroskopiert wurde nach 4 Tagen der Aufbewahrung bei $10^{\circ}$.

\begin{tabular}{|c|c|c|c|c|c|}
\hline \multirow{2}{*}{$\begin{array}{c}\text { Hefesendung } \\
\text { für } \\
\text { Homogenisat }\end{array}$} & Art & \multicolumn{4}{|c|}{ Zahl der Ansätze } \\
\cline { 2 - 6 } & & $\begin{array}{c}\text { A } \\
\text { mit Einsaat }\end{array}$ & \multicolumn{2}{c|}{ ohne Einsaat } \\
\cline { 2 - 6 } & & $\begin{array}{c}\text { ge- } \\
\text { samt }\end{array}$ & positiv & ge- \\
samt & positiv \\
\hline I & Y & 7 & 7 & 8 & 0 \\
II & Z & 7 & 6 & 7 & 0 \\
III & Y & 5 & 5 & - & - \\
& Z & 5 & 5 & - & - \\
& X & 5 & 5 & 15 & 1 \\
& & - & - & 20 & 0 \\
\hline
\end{tabular}

Tab. 6. Prüfung des Homogenisats auf Zellen.

Mit den Kulturen aus der Einsaat zeigt Tab. 6, daß im Homogenisat $\mathrm{Z}, \mathrm{X}$ und $\mathrm{Y}$ zu wachsen vermögen; ferner erlaubt sie den Schlu $\beta$, daß diese Arten nicht schon in diesen Homogenisaten vorhanden waren. Allerdings enthielt einer der 50 Leeransätze nach 4 Tagen eine Kultur, und zwar die Art L. Es ist nicht zu entscheiden, ob sie sich im Homogenisat schon vorfand oder ob nicht ausreichend totverdünnt war.

6. Schärfere statistische Kriterien besitzt ein weiteres Verfahren: Die Anzahl sehr weniger Zellen in gegebenen Volumen läßt sich durch Zerlegung in gleiche Teilvolumina recht genau bestimmen (s. o.).

Für 100 Teilvolumina errechnen sich bei einer Gesamtzahl von 300 Zellen 95, von 100 Zellen 64 zellhaltige. 
R. GAUde verteilte Mischungen von Homogenisat, Zellen B, Hefekochsaft, Glucose und Äthanol in Serien von je $8 \mathrm{ml}$ auf lufthaltige Ampullen, verschmolz und schüttelte 2 bis 3 Tage bei $24,5^{\circ}$. Jede Ampulle enthielt $20 \mu \mathrm{l}$ Homogenisat; auf 50 Ampullen entfielen nach Rechnung 150 oder 50 Zellen. Tab. 7 gibt das Ergebnis wieder.

\begin{tabular}{|c|c|c|c|c|c|}
\hline \multirow{2}{*}{$\begin{array}{c}\text { Zellen pro } \\
\text { 50 Ampullen }\end{array}$} & $\begin{array}{c}\text { Serien } \\
\text { (Homo- } \\
\text { genisate) }\end{array}$ & $\begin{array}{c}\text { Ampullen } \\
\text { insgesamt }\end{array}$ & Zahl & \multicolumn{3}{|c|}{ Prozent } \\
\cline { 5 - 7 } & & & & gef. & ber. \\
\hline 150 & 9 & 95 & 91 & 96 & 95 \\
50 & 5 & 80 & 44 & 55 & 64 \\
\hline
\end{tabular}

Tab. 7. Nachweis weniger Zellen in der Schüttelkultur

Nach Tab. 7 ist die statistische Voraussage der Zahl zellhaltiger Ampullen gut erfüllt. Der Umstand, daß die Zahl in der zweiten Zeile von der Berechnung nach unten abweicht, spricht bereits gegen Zellen im frischen Homogenisat. Der Versuch wurde aber ohne Einsaat und mit wechselnder Menge Homogenisat aus 12 Hefesendungen fortgesetzt und ergab Tab. 8 (21.12. 1961 bis 23.5.1962).

\begin{tabular}{|c|c|c|}
\hline $\begin{array}{c}\text { Homogenisat } \\
{[\mu l \text { pro Ampulle }]}\end{array}$ & $\begin{array}{c}\text { Ampullen } \\
\text { insgesamt }\end{array}$ & $\begin{array}{c}\text { zellhaltige } \\
\text { Ampullen }\end{array}$ \\
\hline 20 und 30 & 136 & 0 \\
40 & 48 & 1 \\
50 und 60 & 30 & 0 \\
\hline
\end{tabular}

Tab. 8. Prüfung des Homogenisats auf Zellen.

Wir entnehmen der Tab. 8, daß alle Proben des Homogenisats bis auf eine leer waren. Diese eine enthielt sehr wenige Zellen einer uns unbekannten Art.

Wird es nach diesen Ergebnissen für praktisch ausgeschlossen gehalten, daß die zahlreich beobachteten Zellkulturen auf Zellen im frischen Homogenisat zurückgehen (und ist Sterilität der Geräte hinreichend gesichert; s. Methodik), so ist noch an Zellen aus der Luft zu denken.

\section{f) Prüfung auf Infektion}

1. Die Schüttelansätze zu Tab. 4 begannen am 14. 12. 1962. Am 20.12. stellte C. Böнme 53 Schälchen mit $3 \mathrm{~cm}^{2}$ Öffnung im Laboratorium auf und spülte durchschnittlich im Abstand von 2 Tagen mit $1,5 \mathrm{ml} \mathrm{Kochsaft/Glucose} \mathrm{aus.} \mathrm{Bis} \mathrm{zum} \mathrm{16.3.} 1963$ gingen in den unter Luft geschüttelten Proben (1 von 1,5 ml) insgesamt 11 Kulturen an, die Arten B, Z und Y.
Die Reduktion der Zellenzahlen ergab öfters $A_{0}<1$; der Grund dafür liegt hier darin, daß bei lufttrockenen Zellen, die in Nährlösung eingetragen werden, die neue Sprossung der einzelnen sich über Stdn. verteilt. So fanden wir es mit B, Z, X und Y.

Nun waren in eben diesen Wochen die Kulturen der Schüttelansätze in 17 Stdn. bis zur Zählbarkeit angewachsen. Sollen sie mit Infektion aus der Luft erklärt werden, dann sind zum Vergleich jene Zellen aus den 53 Schälchen heranzuziehen, die diese Bedingung erfüllen. Aber lediglich in 3 von den 11 Fällen dauerte es nur 20 bis 22 Stdn., bis die ersten Zellen nachweisbar bzw. zählbar waren.

Das erste Schälchen mit Zellen, das achte in der Reihe, hatte 28 Tage offen gestanden. In den Schüttelansätzen aber, zu denen die Luft jeweils nicht länger als 2 Min. direkten Zugang hatte, erschienen 126 Kulturen (Tab. 4).

2. Alle von uns beobachteten Zellarten vermehren sich in Kochsaft von Bäckerhefe. Wir exponierten $85315 \mu$ l-Tropfen von Kochsaft und Schalen mit Kochsaft, in denen dessen Oberfläche 1500 Tropfen entsprach, für 5 Min. an der Luft und fanden nach 4 Tagen 2 Kulturen, Zellen B und nicht identifizierte.

\section{g) Schüttelversuche mit Nucleasen}

Erhielt das Homogenisat Ribonuclease (RNase) oder Desoxyribonuclease (DNase) oder beides, dann war die Ausbeute an Zellkulturen wesentlich geringer. Im nächsten Versuch (Tab.9) sind die Fermente in abgestufter Menge als Zusatz zum Homogenisat und zur Hefe vor der Zerreibung anwesend. Die Ansätze (aus 3 Hefesendungen) entsprechen denen zu Tab. 4, enthalten aber Wasser statt Kochsaft.

Der in Tab. 9 sichtbare Einfluß der Nucleasen wird noch deutlicher durch die Betrachtung der Zellenzahlen: Die auf $t=0$ Min. reduzierten Zahlen $A_{0}$ betrugen im Mittel bei den Kontrollen 3,5, bei den DNase-Ansätzen 0,5. Man könnte die Depression der Kulturen durch $56 \gamma$ DNase näherungsweise mit

angeben.

$$
\frac{31}{3} \cdot \frac{3,5}{0,5} \cdot \frac{23}{24}=\frac{100}{1,45}
$$

Die Wirkung der Nucleasen beruht nicht auf einer Hemmung des Wachstums gegebener Zellen: Die Arten B, Z, A, X und Y wurden mit Kochsaft/Glucose je auf die Konzentration ca. 200 Zellen/ $\mu$ l gebracht und unter Schütteln vermehrt. Parallelansätze enthielten zusätzlich RNase und DNase in der Maxi- 


\begin{tabular}{|c|c|c|c|c|c|c|}
\hline & \multirow{3}{*}{ ohne Nuclease } & \multirow{2}{*}{\multicolumn{2}{|c|}{$\begin{array}{c}\text { RNase } \\
140 \gamma / \text { Ansatz }\end{array}$}} & \multicolumn{2}{|c|}{ DNase } & \multirow{2}{*}{$\begin{array}{c}\text { RNase + DNase } \\
140 \text { bzw. } 56 \gamma\end{array}$} \\
\hline & & & & $14 \gamma$ & $56 \gamma$ & \\
\hline & & zum Homog. & zur Hefe & \multicolumn{3}{|c|}{ zur Hefe } \\
\hline $\begin{array}{l}\text { Ansätze } \\
\text { Kulturen } \\
\quad \text { Arten }\end{array}$ & $\begin{array}{c}24 \\
23 \\
\mathrm{~B}, \mathrm{Z}, \mathrm{A}, \mathrm{X}, \mathrm{L}\end{array}$ & $\begin{array}{r}16 \\
7 \\
\mathrm{Z}, \mathrm{A}\end{array}$ & $\begin{array}{c}10 \\
2 \\
\text { P oder B }\end{array}$ & $\begin{array}{c}26 \\
12 \\
\text { P oder B, X }\end{array}$ & $\begin{array}{c}31 \\
3 \\
Z\end{array}$ & $\begin{array}{r}30 \\
1 \\
Z\end{array}$ \\
\hline
\end{tabular}

Tab. 9. Erniedrigung der Ausbeute an Zellkulturen durch Nucleasen.

malkonzentration nach Tab. 9. Die Fermente erhöhten die Verdoppelungszeiten (Tab. 1) nicht.

Nucleasen bewirken in unserem System die Herabsetzung der Zahl von Zellkulturen. Ihre spezifische Wirkung ist aber die Spaltung freier Nucleinsäuren. Der Schluß liegt nahe, daß die eine Wirkung die Folge der anderen ist, daß also hier - wie bei den atmenden Teilchen ${ }^{1}$ - Zellen gebildet wurden, und daß für ihre Bildung freie Nucleinsäuren anwesend sein müssen.

\section{h) Versuche mit Rhodotorula}

Wir haben unser Verfahren auch auf rote Hefe angewandt. Diese unterscheidet sich von Bäckerhefe u. a. durch minimale Gärung und hat für uns den Vorteil, daß wir sie leicht in Reinkultur gewinnen ${ }^{1}$.

Für folgenden Versuch (Tab. 10) wurde nach dem zweiten Verfahren (vgl. Methodik) homogenisiert und im übrigen so gearbeitet, wie es unter c), 4 . beschrieben wurde. Jedoch waren die Tropfen nur $5 \mu \mathrm{l}$ groß, und je $10 \mu \mathrm{l}$ Homogenisat wurden in die 20 Kontrollampullen gebracht.

Die Tab. 10, für die 10 Zuchten homogenisiert wurden, zeigt, daß bei roter Hefe eine große Zahl von Kulturen auch dann auftrat, wenn die Größe des Tropfens nur $5 \mu \mathrm{l}$ betrug. Dabei ist es wahrscheinlich, daß die häufig auftretenden roten Kulturen oft auf mehr als eine Zelle zurückgingen; wir haben aber rote Hefe im Gefäß immer als eine einzige Kultur gezählt. - Dem Gesamtvolumen der Tropfen $(392 \cdot 5 \mu \mathrm{l})$ entsprechen die Kontrollen $(200 \cdot 10 \mu \mathrm{l})$, die sämtlich frei von Kulturen gefunden wurden.

Wie beim Versuch mit Bäckerhefe erschienen Kulturen, die mit Sicherheit nicht aus der zerriebenen Zellart bestanden (38 von 343 ).
Nun sollte der Vorgang, den wir mit Sand (einem integrierenden Bestandteil des Systems?) in der Reibschale einleiten, auch in der Natur nicht ausgeschlossen sein. Es könnte dann auch dort die eine Art zerstört werden und aus ihren Trümmern die andere Art entstehen. Das wäre keine Mutation.

Wir danken der Deutschen Forschungs gemeinschaft für die Förderung dieser Untersuchung und Frau U. Forstmeyer und Frau M. Dussa für ihre wertvolle Hilfe bei der Ausführung der Versuche.

\section{Methodik}

\section{a) Zellarten}

Zellenzahlen haben wir in der Zählkammer nach BürRER bestimmt. War im Verlauf der Vermehrung der Prozentsatz der sprossenden Zellen konstant, dann konnte die Zählung zur Bestimmung der Verdoppelungszeit $\left(t_{\mathrm{v}}\right)$ dienen.

Die Größenangaben in Tab. 1 werden ergänzt durch das Volumen, das $10^{8}$ Zellen einer Art im geeichten Hämatokriten einnehmen. Wir zentrifugieren ca. $1 \mathrm{ml}$ Suspension $10 \mathrm{Min}$. bei $1200 \mathrm{~g}$ und fanden für B 15 ; Z $18 ; \mathrm{X} 1,5 ;$ Y $8 \mu 1 / 10^{8}$ Zellen.

Gärung und Atmung in 3-proz. Glucoselösung wurden in der W a rburg-Apparatur bei $28^{\circ}$ gemessen. Der Errechnung der Quotienten $Q_{\mathrm{CO}_{2}}^{\mathrm{N}_{2}}$ und $Q_{\mathrm{O}_{2}}$ liegt bei Bäckerhefe das Trockengewicht zugrunde. Für die anderen Zellarten ist unterstellt, daß bei ihnen das gleiche Verhältnis zwischen Trockengewicht und Feuchtvolumen im Hämatokriten gilt.

Für die Bestimmung der Verdoppelungs-Geschwindigkeit bei gärenden Zellen ist die Gärungsgröße nach einer Vorperiode von mehreren Stdn. praktisch und genau. In der Schüttelkultur unter Luft bei $28^{\circ}$ ergab sich mit dem Nährgemisch $0,6 \mathrm{ml}$ Kochsaft, $0,2 \mathrm{ml}$ 20-proz. Glucose, $0,2 \mathrm{ml}$ Zellsuspension in Wasser für Bäckerhefe $t_{\mathrm{v}}=100$ Min. (6). Bei unterschiedlichen

\begin{tabular}{|c|c|c|c|c|c|c|c|c|c|c|}
\hline \multirow{2}{*}{$\begin{array}{l}\text { Zahl der } \\
\text { Tropfen }\end{array}$} & \multicolumn{5}{|c|}{ Kulturen } & \multirow{2}{*}{ Mischkult. } & \multicolumn{2}{|c|}{ Kontrollen } & \multicolumn{2}{|c|}{ Gemisch-Ampullen } \\
\hline & $\mathrm{Z}$ & A & $\mathrm{S}$ & $\mathrm{Y}$ & SS & & positiv & negativ & positiv & negativ \\
\hline 392 & 5 & 2 & 5 & 305 & 26 & 33 & 0 & 200 & 0 & 30 \\
\hline
\end{tabular}

Tab. 10. Zellen aus Homogenisat von roter Hefe. 
Ernährungsbedingungen fanden wir lediglich folgende Abweichungen: Unter Stickstoff 101 Min. (6); unter Stickstoff mit und ohne Phosphor im Einsatz und mit Homogenisat aus Bäckerhefe (statt Kochsaft) 104 Min. (31) ; unter Luft mit Nährlösung nach OLson und JoHNson ${ }^{2}$ (statt Kochsaft) 107 Min. (13); unter Luft bei $24,5^{\circ}$ mit Homogenisat 101 Min. (4).

Nicht alle Zellarten, die wir mikroskopisch unterscheiden konnten, sind in Tab. 1 enthalten; seltener vorkommende haben wir nicht charakterisiert.

\section{b) Homogenisate}

Es wurden Bäckerhefe der Firma Sinner, Karlsruhe, und Rhodotorula eigener Zucht (vgl. 1. Mitt.) verwandt. Das erste Zerreibungsverfahren wurde oben beschrieben, die Vorschrift für das zweite ist folgende: Sogleich nacheinander werden je 1,2 g feuchte Hefe, 0,1 ml Wasser und $2 \mathrm{~g}$ Sand in der Reibschale so stark verrieben, daß das Gemisch in 3,5 Min. eben noch nicht trocken geworden ist. Der Inhalt der ersten Schale wird mit $11,8 \mathrm{ml}$ Wasser und $2 \mathrm{ml} \mathrm{20-proz.} \mathrm{Glucose} \mathrm{in} \mathrm{die} \mathrm{zweite}$ überspült. Bei $0^{\circ}$ und $10000 \mathrm{~g}$ wird zentrifugiert, und zwar die sandhaltige Suspension 10 Min., ihr Überstand 15 Min., der zweite Überstand 20 Min. Von dem dritten Überstand wird oben ca. $1 \mathrm{ml}$ mit der Injektionsspritze abgehoben.

Alle in dieser Arbeit verwandten Geräte aus Glas, Metall oder Ton sind nach der Reinigung in kochendes Wasser gebracht und/oder feucht für mindestens $3 \mathrm{Stdn}$. auf $120^{\circ}$ gebracht worden. Alle Geräte, die für die Versuche mit Homogenisat nach dem zweiten Verfahren in Berührung kamen, waren feucht für mindestens $3 \mathrm{Stdn}$. auf $200^{\circ}$ gebracht worden und wurden jeweils für den Gebrauch dem abgekühlten Trockenschrank entnommen. Alle für die Herstellung von Homogenisat und für Versuche verwandten Flüssigkeiten, einschließlich Wasser, stammten aus ein- oder zweimal für $2 \mathrm{Stdn}$. auf $115^{\circ}$ erhitzten Ampullen, die vor dem Aufbrechen abgeflammt waren.

${ }^{2}$ B. H. Olson u. M. J. Johnson, J. Bacteriol. 57, 235 [1949].

\section{c) Tropfenmethoden}

1. Die Schale hat $18 \mathrm{~cm}$ Durchmesser und $6 \mathrm{~cm}$ Höhe. Der Deckel besitzt 3 kleine Löcher, die vor dem Sterilisieren mit 4 Lagen Zellstoff verklebt werden. Die 40 Schälchen haben $2 \mathrm{~cm}$ Durchmesser und $1 \mathrm{~cm}$ Höhe. Meist $300 \mathrm{mg}$ körniges Calciumchlorid lagen beim Sterilisieren schon im zentralen Gefäß. Für das Einbringen des Homogenisats wird eine Pipette mit gemessener Tropfengröße durch den Zellstoff gestochen und unter Drehung des Deckels von Schälchen zu Schälchen bewegt. Auch zur Bewässerung des Bodens und des Calciumchlorids wird ein Verschluß durchstoßen. Mit Gummistopfen wird wieder verschlossen.

Um den Verlauf der Verdunstung und der Redestillation von Wasser in der Schale zu verfolgen, haben wir die elektrische Leitfähigkeit eines der Tropfen gemessen und eine Federwaage aus Glas benutzt, die zwischen 0,4 und $6 \mathrm{mg}$ auf $10 \%$ genau anzeigt. Wir fanden, daß $15 \mu \mathrm{l}$ Homogenisat gegen $0,6 \mathrm{mg}$ zurückgingen und Bewässerung ca. 1,6 mg erbrachte. 1,8 mg entsprechen ungefähr der Plasmakonzentration.

2. Die Tropfen erhielten nach 3 Tagen $0,1 \mathrm{ml}$ von dem Gemisch 3 Vol. Kochsaft, 1 Vol. 20-proz. Glucose, 4 Vol. Wasser, darin 0,15\% Streptomycin „Bayer“. Streptomycin hat keinen Einfluß auf die Verdoppelungszeit roter Hefe. Bakterielle Infektion trat in den oben beschriebenen Versuchen recht selten ein und hat die Bestimmung der Hefezellen nicht gestört. Aber bei Vorversuchen, in denen die Zimmerluft leichten Zutritt hatte, war das Bacteriostaticum nützlich, und wir behielten es bei.

\section{d) Schüttelversuche mit Nucleasen}

RNase aus Rinderpankreas war ein Produkt der Firma Mann, New York, N. Y., DNase eines der Firma Worthington, Freehold, N.J.; beide waren mehrfach kristallisiert und salzfrei.

Die Unempfindlichkeit der Zellvermehrung gegen Nucleasen im Milieu wurde für die Art A $\left(t_{\mathrm{v}}=\right.$ 140 Min.) durch Atmungsmessung erkannt. Die Gefäße a (ohne) und b (mit Nucleasen) atmeten zu Beginn beide mit $-3,6 \mu \mathrm{l} \mathrm{O}_{2} / 10 \mathrm{Min}$; nach $487 \mathrm{Min}$. atmete a mit -32 , b mit $-33 \mu \mathrm{l} / 10$ Minuten. Aus diesen Daten folgt $t_{\mathrm{v}}=152$ Minuten. 